\title{
Recommendations for neonatologist performed echocardiography in Europe: Consensus Statement endorsed by European Society for Paediatric Research (ESPR) and European Society for Neonatology (ESN)
}

\author{
Willem P. de Boode ${ }^{1}$, Yogen Singh ${ }^{2}$, Samir Gupta ${ }^{3}$, Topun Austin ${ }^{4}$, Kajsa Bohlin ${ }^{5}$, Eugene Dempsey ${ }^{6}$, Alan Groves ${ }^{7}$, \\ Beate Horsberg Eriksen ${ }^{8}$, David van Laere ${ }^{9}$, Zoltan Molnar ${ }^{10}$, Eirik Nestaas ${ }^{11,12}$, Sheryle Rogerson ${ }^{13}$, Ulf Schubert ${ }^{14}$, Cécile Tissot ${ }^{15}$, \\ Robin van der Lee ${ }^{16}$, Bart van Overmeire ${ }^{17}$ and Afif El-Khuffash ${ }^{18,19}$
}

\section{INTRODUCTION}

The use of echocardiography for the evaluation of the cardiovascular well-being of term and preterm infants is gaining significant interest. The aim of echocardiography in this setting is to provide hemodynamic information in real time in order to support bedside clinical decision making (1-3). This approach is perceived to enhance clinical judgment, provide a better understanding of active physiological processes, and monitor the response to treatment. Combination of clinical examination and bedside echocardiography has been shown to facilitate clinical decision making (4). There is some evidence that routine use of echocardiography on the neonatal unit might lead to early identification of cardiovascular compromise that could facilitate clinical management (5), potentially improving short-term outcomes $(6,7)$. There is growing literature highlighting the potential merits of echocardiography in the identification of cardiovascular compromise and guiding neonatal cardiovascular care $(4,6,8,9)$.

There is a need to ensure standardization of training and clinical practice guidelines, with quality assurance systems in place to maintain safe dissemination of this practice. There are currently two published guidelines in neonatal echocardiography with recommendations for training and an accredited structured training program with accreditation in neonatal cardiac ultrasound. The practice guidelines and recommendations for the use of echocardiography (Targeted Neonatal Echocardiography (TNE)) and Expert consensus statement on Neonatologist performed echocardiography (NoPE) in the United Kingdom were published in the last 5 y $(10,11)$.
However, some aspects of these two guidelines are difficult to implement in a pan European setting. The Australian Society of Ultrasound in Medicine has developed and implemented a basic training and accreditation process in Australia encompassing cardiac, brain, and abdominal ultrasound that is difficult to adopt in Europe (http://www.asum.com.au).

As a result, a working group under the auspices of the European Society for Paediatric Research and the European Society for Neonatology was convened to devise a consensus statement for neonatologist performed echocardiography training taking into account the heterogeneous nature of training facilities, personnel, and infrastructure across Europe. In this statement, we aim to (i) highlight the difficulties in implementing the current TNE and UK guidelines in a European setting; (ii) define the training facilities and infrastructure necessary for optimal training conditions; (iii) identify training standards applicable across Europe; and (iv) discuss some practical aspects including a suggested governance structure for oversight of training and continued quality assurance. We have elected to use the term neonatologist performed echocardiography (NPE) as used in the UK guideline because it identifies the person performing the assessment and the target organ assessed.

This consensus statement deals with NPE for assessment of hemodynamic function in infants with a structurally normal heart (patent ductus arteriosus (PDA) and patent foramen ovale included). Although confirmation of normal structural anatomy is within the remit of this practice, diagnosis and management of congenital heart disease (CHD) is beyond the scope of this guideline. This practice should always remain

\footnotetext{
'Department of Neonatology, Radboud University Medical Center, Nijmegen, The Netherlands; ${ }^{2}$ Addenbrooke's Hospital, Cambridge University Hospitals NHS Foundation Trust, Cambridge, UK; ${ }^{3}$ University Hospital of North Tees, Durham University, Stockton-on-Tees, UK; ${ }^{4}$ Department of Neonatology, Rosie Hospital, Cambridge University Hospitals NHS Foundation Trust, Cambridge, UK; ${ }^{5}$ Department of Neonatology, Karolinska University Hospital, Karolinska Institutet, Stockholm, Sweden; 6 INFANT Centre, Cork University Maternity Hospital, University College Cork, Cork, Ireland; ${ }^{7}$ Weill Cornell Medical College New York, New York, New York; ${ }^{8}$ Department of Pediatrics, Møre and Romsdal Hospital Trust, Ålesund, Norway; ${ }^{9}$ Department of Pediatrics, Antwerp University Hospital UZA, Edegem, Belgium; ${ }^{10}$ Department of Physiology, Anatomy and Genetics, John Radcliffe Hospital, Oxford, UK; ${ }^{11}$ Institute of Clinical Medicine, Faculty of Medicine, University of Oslo, Oslo, Norway; ${ }^{12}$ Department of Cardiology and Center for Cardiological Innovation, Oslo University Hospital, Rikshospitalet, Oslo, Norway; ${ }^{13}$ Department of Neonatology, The Royal Women's Hospital, Parkville, Victoria, Australia; ${ }^{14}$ Department of Clinical Science, Intervention and Technology, Karolinska Institutet, Stockholm, Sweden; ${ }^{15}$ Pediatric Cardiology Unit, Geneva University Children's Hospital, Geneva, Switzerland; ${ }^{16}$ Department of Neonatology, Emma Childrens' Hospital AMC, Amsterdam, The Netherlands; ${ }^{17}$ Department of Neonatology, University Hospital Brussels, Brussels, Belgium; ${ }^{18}$ Department of Neonatology, The Rotunda Hospital, Dublin, Ireland; ${ }^{19}$ School of Medicine (Department of Pediatrics), The Royal College of Surgeons in Ireland, Dublin, Ireland. Correspondence:Willem P.de Boode (willem.deboode@radboudumc.nl)
} 


\section{Special Article}

under the remit of pediatric cardiology services. Preterm and term infants with clinical or echocardiographic suspicion of CHD should be immediately referred to a pediatric cardiologist.

\section{THE CURRENT GUIDELINES IN A EUROPEAN CONTEXT}

Neonatology services in Europe are very heterogeneous in nature and include Level 1 and 2 units, stand-alone Level 3 maternity units, those colocated with adult services, and quaternary maternity units located either physically with, or in the vicinity of, a children's hospital. More importantly, the proximity between pediatric cardiology services and neonatal intensive care units (NICUs) are very variable across Europe. In general, pediatric cardiology services in Europe are under pressure and might not be adequately resourced to take in an additional influx of echocardiography trainees outside a dedicated cardiology fellowship program. As a result, a "one size fits all" approach for NPE training and accreditation in Europe may not be feasible.

The American Society of Echocardiography/European Association of Echocardiography/Association for European Paediatric Cardiology guideline for TNE training is an ideal example of a structured approach to standardize training and maintenance of competence $(11,12)$. Our consensus group fully endorses large sections of those guidelines including the background and indications, practical aspects, the suggested approach to the various clinical indications (Sections 1 and 2), and we suggest the reader to refer to the sections for a comprehensive overview of those topics. However, certain aspects of the proposals for training are currently not achievable in a European context. The guideline proposes a two-phase process of a core training period followed by an advanced period of training, both lasting 4-6 mo. During the core period, the candidates should carry out $>150$ scans and review a further 150 scans, $80 \%$ of which should be structurally abnormal. This training is mandated to be carried out in an echocardiography laboratory under the direction of the pediatric cardiology service. Following an unspecified assessment process, the candidate moves on to the advanced stage for another period of 4-6 mo and an additional 150 scans and 150 study reviews. The suggested place of training is the NICU for the advanced phase. However, direct or indirect supervision and coordination by the pediatric cardiology service are mandated during this phase of training as well.

This approach to training has faced difficulties in lead North American centers and is not feasible in Europe where pediatric cardiology services cannot accommodate a significant influx of neonatal trainees into their already saturated echocardiography laboratories. We suggest that NPE training would define the standards to be met while delivered through a close collaboration between neonatologists and pediatric cardiologists. Training in an echocardiography laboratory for the neonatal trainee may be an alternative option where training cannot feasibly be conducted in an NICU setting. Eventually Neonatology fellowship programs in Europe should incorporate echocardiography training, but in the interim an intermediate model for acquiring competencies will be suggested. Similarly, consultant neonatologists planning to undertake NPE may not be able to take a 6-mo period away from clinical practice, but will be required to meet the standards and demonstrate engagement so alternate solutions need to be determined.

The Expert Consensus Statement for NoPE training and accreditation in the United Kingdom (three of whom are also authors in this statement: Y.S., S.G., and A.G.) is endorsed by Neonatologists in the United Kingdom with interest in Cardiology and Haemodynamics and the British Congenital Cardiac Association, and they have tailored training to meet the requirements without compromising on the quality. The guidelines mandate a 6-mo period of training to be carried out in a pediatric cardiology department during core registrar or higher specialist training in neonatology (10) and another minimum of 6 mo in a neonatal center with expertise in echocardiography for hemodynamic assessment. It provides guidance for new trainees, those who are already practicing, and for maintaining competencies. While this approach is appropriate in a UK setting, for reasons outlined above, this might be difficult to be implemented in some parts of Europe where pediatric cardiology placements might not be available.

Other European countries have established their own training guidelines. In Switzerland, a working group with representatives of the Swiss Society of Neonatology and the Swiss Society of Paediatric Cardiology has adapted the European recommendations on TNE, with two levels of training: core and advanced (http://www.neonet.ch/en/education/ postgraduate-training-neonatology).

In Australia, a Neonatal Certificate in Clinician Performed Ultrasound provides the only accredited training pathway currently in existence for cardiac, brain, and abdominal ultrasound. The Australian Society of Ultrasound in Medicine should be commended for developing and instituting this program. This program operates on the premise that $\mathrm{CHD}$ has been excluded by a pediatric cardiologist or an equivalently trained person, which is generally done by review of the initial scan by the nominated expert. After a 2-d basic course, the trainees have to be signed off by a supervisor after doing 50 cardiac and 25 head scans. After approval of the log book and passing a physics course, the trainee qualifies for basic Certificate in Clinician Performed Ultrasound and is eligible to attend the advanced course. After completion of a 2-d advanced course, an additional 50 cardiac scans, 20 cranial scans, and 10 abdominal scans should be performed. As this program does not require establishing structural normality of heart and includes other organ scans, it cannot be adopted as it is. It requires modification to allow integrating in clinical practice and develop a system, which is fit for practice across Europe where emphasis is on confirming structural normality.

\section{TRAINING FACILITIES AND INFRASTRUCTURE}

We advocate that NPE training in Europe could be carried out in a neonatal intensive care setting with appropriate supervision by pediatric cardiologists or accredited neonatologists 
with expertise in echocardiography. In cases where this is not feasible, initial training can occur through a pediatric cardiology program with emphasis on neonatal pathology. We advocate that training should be coordinated, directed, and conducted by a qualified neonatologist with the necessary skillset (see Credentials of NPE trainers). However, we also suggest that endorsement by, and close collaboration with, local pediatric cardiology services is an absolute necessity for a successful training program. In the section below we will outline the necessary infrastructure and personnel requirements needed to run a successful training program in a European Setting.

\section{Training in a Tertiary/Quaternary NICU or Pediatric Cardiology Center}

Tertiary units undertaking NPE training should fulfil certain criteria which are intended to ensure that NPE trainees will be exposed to a wide variety of gestations and pathologies including exposure to $\mathrm{CHD}$ during the early neonatal period:

- The unit should be performing regular echocardiography (more than five scans per week), admit infants $<1,500 \mathrm{~g}$, be a center for therapeutic hypothermia, and provide therapeutic modalities which include high-frequency ventilation and inhaled nitric oxide.

- The NICU should admit infants with suspected or confirmed CHD prior to transfer to pediatric cardiology services. This will facilitate planned scanning of those infants for learning purposes under the guidance of a pediatric cardiologist.

- Close collaboration with pediatric cardiology services is essential and should include regular onsite visit of a consultant pediatric cardiologist.

- The unit should be an accredited training site with the local body tasked with neonatology fellowship training.

Emphasis should be placed on infants with normal structural anatomy but with neonatal hemodynamic pathophysiology. We do not recommend that formal NPE training be undertaken in Level I and II units as those units will lack an adequate mix of pathologies and extremely preterm infants.

\section{Credentials of NPE Trainers}

As stated above, directing, coordinating, and conducting training should be done by neonatologists with an extensive expertise in NPE. Currently, no formal certification in NPE for trainer or trainees exists. Therefore, NPE trainers should possess a skill set that is sufficient for independent scanning and facilitates training: Trainers should be able to obtain all the required echocardiography views (in B-mode (2D), M-Mode, and color Doppler), be competent in confirming normal structural anatomy, identifying pathology, and be fully competent in performing all the functional modalities outlined in the TNE/NoPE guidelines $(11,12)$. Familiarity and knowledge of advanced functional techniques including tissue Doppler imaging and speckle tracking echocardiography is not essential but is desirable. Other key elements should include the following:

- A 12-mo period of exposure to neonates with CHD under the supervision of a designated consultant pediatric cardiologist. This period can be feasibly carried out in a tertiary neonatal center fulfilling the criteria outlined above. Although time in a pediatric heart center is not essential, it may be of great benefit for further exposure to CHD.

- NPE trainers should have a designated and established link with a consultant pediatric cardiologist who endorses the echocardiography skills of the trainer and should maintain a logbook of scans and report on a standard template. Regular meetings to discuss cases and review trainee progress with pediatric cardiology are essential.

- NPE trainers should carry out at least 50 echocardiograms per annum themselves to demonstrate maintenance of competency. Those scans should be functional in nature in structurally normal hearts and not for the purpose of diagnosis of CHD. We understand that some neonatologists across Europe do perform echocardiography to rule out $\mathrm{CHD}$, but this practice is beyond the scope of this consensus statement.

- NPE trainers should have dedicated time allocated to training oversight on a weekly basis.

\section{Echocardiography Equipment and Archiving}

Echocardiography equipment used for training purposes should have B-mode (2D), M-mode, pulsed wave, continuous wave, and color Doppler facilities. Tissue Doppler imaging capability is not a prerequisite for training. Electrocardiogram tracing capability is also an essential component that should be used on all scans. A range of probes should be available to cater for a wide range of infant sizes. A dedicated work station with an archiving system for image storage and offline review in the vicinity of the NICU is required to facilitate study storage, review, and reporting. Reporting of echocardiography examinations should be formalized and standardized. Studies undertaken for teaching purposes should be reported as such.

\section{GOVERNANCE STRUCTURE}

Currently an overarching governance structure for NPE across Europe does not exist. However, the ultimate aim is to form a pan European Neonatal Echocardiography Certification Program with close collaboration with the Association for European Paediatric Cardiology. This program will be run by neonatologists with advanced echocardiography skills in collaboration with pediatric cardiologists and will serve to assess local training structures and provide accreditation, training oversight, and direct continuing assessment. This program should be endorsed by the European Society for Paediatric Research and the European Society for Neonatology. In the interim we propose that unit which fulfil the requirements 


\section{Special Article}

for training outlined above should nominate an advanced NPE trainer to act as a director for the local training program and identify a pediatric cardiologist who will act as training support. The units considering training should adhere to the training requirements set out below.

Regular echocardiography imaging rounds should be part of the training structure. This will complement learning and maintain quality standards.

\section{TRAINING REQUIREMENTS}

Introduction to Echocardiography Concepts (Pretraining Phase) Candidates undertaking an NPE training program should partake in a pretraining phase aimed to prepare the candidate for carrying out echocardiograms. A thorough and comprehensive understanding of cardiovascular anatomy, physiology, and hemodynamics, as it applies to neonatal health and disease, is essential as NPE should always be taken in the clinical context and used to guide clinical management. In addition, the candidate should demonstrate an understanding of the physics and principles of ultrasound, pulsed wave, continuous wave, and color Doppler. In order to aid in image acquisition during the practical training phase, the candidate should become familiar with all the necessary echocardiography views required to perform a standard NPE study including $\mathrm{M}$-mode imaging and its use in functional echocardiography. There are a variety of print and online multimedia resources available to facilitate this pretraining phase (Table 1). Attendance at an echocardiography course is also encouraged. The use of echocardiography simulators when available may also be a valuable aid to learning.

\section{Basic Echocardiography Training}

By the end of the basic NPE training phase, the candidate should be able to demonstrate competent operation of the echocardiography machine and should have the ability to use appropriate probes, optimize images, and adequately store and archive completed studies. In addition, the candidate should be capable of adequately acquiring all of the basic echocardiographic views, perform pulsed wave, continuous wave, and color Doppler interrogation as appropriate, use M-mode echocardiography to measure basic function and wall dimensions, and be capable of demonstrating normal structural anatomy of the heart.

Table 1. Supportive material for NPE training

Resources available for NPE pretraining

Echocardiography for the Neonatologist, 1st Edition by Jonathan Skinner MBChB DCH MRCP(UK) FRCPCH MD (Editor), Dale Alverson MD (Editor), Susan M Hunter BA Hons PGDips. ISBN-13: 978-0443054808

http://www.tnecho.com

http://www.neonatalechoskills.com

https://practical-neonatal-ultrasound.selz.com

https://itunes.apple.com/ie/app/tnecho

NPE, neonatologist performed echocardiography.
The candidate should be able to communicate those findings to an NPE trainer and compose a written report of the findings. The candidate should not independently utilize NPE to guide clinical decisions during the basic training phase. Following completion of the basic training, the echocardiograms performed by the candidate can be used to guide clinical management once reviewed by an NPE trainer. We emphasize on strengthening clinical governance by reviewing these studies in departmental echocardiography meetings, which should be led by an advanced NPE trainer. Echocardiography simulators can be very valuable in the early part of training, especially for the assessment of basic echocardiographic views, without having to interfere with the patient's homeostasis. Also the identification of structural abnormalities can be trained on a simulator. It is evident that echocardiography simulators can never replace live patient scanning. Moreover, it is of the upmost importance that good quality simulators are used. It is recommended that available echocardiography models are evaluated prospectively in their feasibility in NPE training. We propose the following key elements that are required to complete the basic echocardiography training phase:

- Complete, log, archive, and report $>100$ complete NPE studies over a 6-mo period. Part of these scans may be performed on a neonatal echocardiography simulator (limited to 50). It is emphasized that the number of a hundred scans is an absolute minimum requirement, and the NPE trainer can decide that a candidate needs more scans to become competent in image acquisition and relating the findings to the clinical scenario, especially if a simulator is used for some of the scans.

- Studies performed during the initial training period while becoming acquainted with the machine and probe positioning do not form part of those 100 studies.

- At least $70 \%$ of those studies should be in infants with normal structural anatomy (including PDA/patent foramen ovale). Infants with a functional pathology, such as PDA, persistent pulmonary hypertension of the newborn, impaired myocardial performance, pericardial effusion et cetera are included in this $70 \%$ group, as are assessments of intravascular catheter position.

- A minimum of 15 of those studies should be in infants with CHD (other than PDA/patent foramen ovale), with at least 10 during the early neonatal period. Those scans should all be reviewed with a pediatric cardiologist or an NPE trainer.

During the basic training phase, the candidate should perform at least five NPE studies observed by the NPE trainer and/ or the pediatric cardiologist to demonstrate competencies. The candidate will be approved to proceed to the advanced training phase when all the training requirements are fulfilled and that the trainee is adequately skilled at the disposal of the NPE trainer. 


\section{Advanced Echocardiography Training}

Following the advanced NPE training phase, the candidate should be able to competently acquire fully optimized images, confidently demonstrate normal structural anatomy, perform complete functional assessment on infants with a wide range of gestations and pathologies including the measurement of dimensions, be able to interpret findings in the clinical content, and devise a management and follow-up plan that is appropriate to the patient. An understanding of the physiology of different hemodynamic conditions is essential. In addition, the candidate should demonstrate an understanding of the limitation of all the echocardiography techniques used. Following the completion of this training the candidate should be able to independently perform NPE scans and use the newly acquired skill in clinical practice. We propose the following key elements that are required to complete the advanced echocardiography training phase:

- Complete, log, archive, and report >100 complete NPE studies including a functional assessment over a 6- to 12-mo period (equating to a minimum frequency of two

Table 2. Approach to confirming normal structural anatomy

\begin{tabular}{|c|c|c|}
\hline Echocardiography view & First study & Subsequent scans \\
\hline $\begin{array}{l}\text { Situs and position of the heart in the } \\
\text { thorax }\end{array}$ & $\sqrt{ }$ & \\
\hline $\begin{array}{l}\text { Systemic venous return to the RA } \\
\text { (IVC/SVC) }\end{array}$ & $\sqrt{ }$ & \\
\hline Left and right atrial size and shape & $\sqrt{ }$ & $\sqrt{ }$ \\
\hline $\begin{array}{l}\text { Intra-atrial septum, PFO, ASD, } \\
\text { direction of shunting }\end{array}$ & $\sqrt{ }$ & $\sqrt{ }$ \\
\hline$A V$ valve morphology & $\sqrt{ }$ & \\
\hline Atrio-ventricular concordance & $\sqrt{ }$ & \\
\hline $\begin{array}{l}\text { Presence of } A V \text { regurgitation/flow } \\
\text { acceleration }\end{array}$ & $\sqrt{ }$ & $\sqrt{ }$ \\
\hline Ventricular chamber size and shape & $\sqrt{ }$ & $\sqrt{ }$ \\
\hline $\begin{array}{l}\text { Presence or absence of VSDs } \\
\text { (Sweeps required) }\end{array}$ & $\sqrt{ }$ & $\sqrt{ }$ \\
\hline $\begin{array}{l}\text { Left and right ventricular outflow tract } \\
\text { obstruction }\end{array}$ & $\sqrt{ }$ & $\sqrt{ }$ \\
\hline Ventricular-arterial concordance & $\sqrt{ }$ & \\
\hline $\begin{array}{l}\text { Aortic and pulmonary valve } \\
\text { morphology }\end{array}$ & $\sqrt{ }$ & \\
\hline $\begin{array}{l}\text { Presence of aortic and pulmonary } \\
\text { valve regurgitation }\end{array}$ & $\sqrt{ }$ & $\sqrt{ }$ \\
\hline $\begin{array}{l}\text { Aortic valve leaflets and coronary } \\
\text { origins }\end{array}$ & $\sqrt{ }$ & \\
\hline Branch pulmonary artery size and flow & $\sqrt{ }$ & $\sqrt{ }$ \\
\hline Presence/absence of PDA and shunt & $\sqrt{ }$ & $\sqrt{ }$ \\
\hline Arch patency & $\sqrt{ }$ & $\sqrt{ }$ \\
\hline $\begin{array}{l}\text { Pulmonary venous drainage into left } \\
\text { atrium }\end{array}$ & $\sqrt{ }$ & \\
\hline
\end{tabular}

ASD, atrial septal defect; AV, atrio-ventricular; IVC, inferior vena cava; PDA, patent ductus arteriosus; PFO, patent foramen ovale; RA, right atrium; SVC, superior vena cava; VSD, ventricular septal defect. scans per week) in the NICU. Up to 50 of these scans may be performed on a neonatal echocardiography simulator, provided that also structural abnormalities and/ or functional measurements can be trained.

- Interpret all the scans in a clinical context and devise a management plan with the NPE trainer.

- Continued exposure to infants with CHD should occur aiming for an additional 10-20 scans in infants with known structural abnormalities. All of those scans should be reviewed with a pediatric cardiologist or an NPE trainer.

Table 3. Summary of recommendations for NPE Training

\section{NPE training centers}

- Level III neonatal intensive care unit

- Accredited site with neonatology fellowship training

- Performing regular echocardiography (more than five scans per week)

- Admission of infants with suspected or confirmed CHD prior to transfer to pediatric cardiology services

- Close collaboration with pediatric cardiology services

\section{NPE trainer}

- 12-mo period of exposure to neonates with CHD under the supervision of a designated consultant pediatric cardiologist

- Designated and established link with a consultant pediatric cardiologist who endorses the echocardiography skills of the trainer

- A minimum of 50 echocardiograms per year (functional in nature)

- Maintenance of a logbook of scans

- Report on a standard template

- Regular meetings to discuss cases and review trainee progress with pediatric cardiology

- Dedicated time allocated to training oversight on a weekly basis

Basic echocardiography training

- Complete, log, archive, and report >100 complete NPE studies over a 6-mo period

- At least $70 \%$ of these studies in infants with normal structural anatomy (including PDA/PFO)

- A minimum of 15 studies in infants with CHD (other than PDA/PFO) with at least 10 during the early neonatal period

- At least five NPE studies observed by NPE trainer and/or pediatric cardiologist to demonstrate competencies

\section{Advanced echocardiography training}

- Complete, log, archive, and report $>100$ complete NPE studies including a functional assessment over a 6- to 12-mo period

- Interpret all scans in a clinical context and devise a management plan

- Additional 10-20 scans in infants with known structural abnormalities, reviewed with a pediatric cardiologist or NPE trainer

Maintenance of competence after advanced training

- At least 50 NPE studies per annum

- Establish a formal link with a designated pediatric cardiologist

- Maintenance of a logbook of scans

- Report on a standard template

CHD, congenital heart disease; NPE, neonatologist performed echocardiography; PDA, patent ductus arteriosus; PFO, patent foramen ovale. 


\section{Special Article}

Following completion of the advanced training phase, the candidate should demonstrate a study with functional assessment in at least one infant with the following conditions: PDA, persistent pulmonary hypertension of the newborn, infant undergoing therapeutic hypothermia, infant with hypotension, and infant with a central line. In addition, the NPE trainer and the designated pediatric cardiologist should assess the candidate's ability to confirm normal structural anatomy of the heart.

To maintain ongoing competency, the successful candidate should continue to perform 50 NPE studies per annum and establish a formal link with a designated pediatric cardiologist as outlined in the trainer requirements above. If the candidate wishes to become an NPE trainer, a time based progression over a 3- to 5-y period demonstrating engagement with pediatric cardiology services, and skill maintenance is required. A further 6 mo training in a cardiac center is encouraged.

\section{PRACTICAL ASPECTS}

\section{Considerations for Scanning Preterm Infants}

Preterm infants are particularly vulnerable to prolonged handling and external stimuli. Therefore, extreme care should be taken when performing NPE in very-low-birth-weight infants. A specific approach to those infants should be considered. We recommend the use of warm sterile ultrasound gel for all imaging to reduce insensible heat loss. The duration of first examination should not be greater than $30 \mathrm{~min}$, and subsequent scans not more than 15 min duration. A clear electrocardiogram signal should be obtained on the imaging screen and time should be taken to ensure the optimal environment for imaging. Measurement of dimensions and function should ideally be done offline following the completion of image acquisition to minimize discomfort. The use of sucrose cannot be recommended routinely but on an ad hoc basis as a recent randomized controlled trial demonstrated no additional benefit and nonpharmacological methods should be attempted first to limit discomfort (13).

\section{The "First Scan" and Normal Structural Anatomy}

One of the principal concepts of healthcare provision is "first do no harm." The use of NPE to guide clinical care should only be carried out once normal structural anatomy is confirmed. Significant potential harm can occur if treatment is instigated in the presence of undiagnosed CHD. Obvious examples include PDA treatment in an undiagnosed ductal dependent lesion, commencing inhaled nitric oxide in infants with total anomalous pulmonary venous drainage, using diuretics in infants with left ventricular outflow tract obstruction, and excessive use of oxygen in infants with undiagnosed left to right shunts (ventricular septal defects). An assumption of the absence of CHD is in our view an unacceptable approach. As a result, the first echocardiogram should be comprehensive enough to reliably confirm normal structural anatomy. We propose an approach to confirming normal structural anatomy in Table 2. Follow-up studies should also examine certain anatomical components to ensure that interventions used did not have any unanticipated effects (e.g., PDA closure and arch constriction).

We propose that a designated NPE trainer can reliably confirm structural anatomy in infants where the clinical suspicion of CHD is low and the recommended approach outlined in Table 2 is followed. A clear distinction should be made between performing an NPE for functional assessment in infants where the level of clinical suspicion of CHD is low, and performing an echocardiogram to rule out $\mathrm{CHD}$ when there is a clinical suspicion of abnormal structural anatomy. The scope of practice of a neonatologist should always be the former. If there is a clinical or echocardiography suspicion of CHD, then the infant should be referred to a pediatric cardiologist. We recognize that many neonatologists across Europe (including several authors of this statement) undertake additional clinical roles in diagnosis and follow-up of CHD. This however should only be done in collaboration with local pediatric cardiology services and is beyond the scope of this consensus statement.

\section{THE USE OF NEWER FUNCTIONAL ASSESSMENT TECHNIQUES}

There has been a recent interest in the use of tissue Doppler velocities and deformation imaging using tissue Doppler and speckle tracking techniques for the assessment of myocardial performance in term and preterm infants (14-20). Those techniques have proven to be valuable diagnostic tools in adults and children with some studies in neonates demonstrating some usefulness in the clinical setting $(14,21)$. In addition, the objective assessment of RV function with quantitative parameters is becoming well established (17,22-26). Although we cannot recommend routine clinical use of those functional techniques, we encourage further exploration of those measurements in the research setting.

\section{CONCLUSION}

NPE is an evolving practice in Europe. The pace of its evolution and uptake by neonatologists are increasing rapidly. However, in order to maintain patient safety and ensure the optimal use of this skill, adherence to training guidelines along with continued collaboration with our pediatric cardiology colleagues is of paramount importance. Given the heterogeneity in training and accreditation throughout Europe, a minimum requirement for NPE is defined (see Table 3), that can serve as a foundation upon which each country can base their national guideline given their specific logistic characteristics.

These recommendations will be evaluated every 3 y by the European Society for Paediatric Research and the European Society for Neonatology working group and where appropriate revised.

\section{STATEMENT OF FINANCIAL SUPPORT}

Part of the accommodation costs during meetings of the Special Interest Group in Neonatologist Performed Echocardiography were supported by the European Society for Paediatric Research (ESPR) and European Society for Neonatology (ESN).

Disclosure: The authors do not have any conflicts of interest to declare. 


\section{REFERENCES}

1. El-Khuffash AF, McNamara PJ. Neonatologist-performed functional echocardiography in the neonatal intensive care unit. Semin Fetal Neonatal Med 2011;16:50-60.

2. Evans N, Gournay V, Cabanas F, et al. Point-of-care ultrasound in the neonatal intensive care unit: international perspectives. Semin Fetal Neonatal Med 2011;16:61-8.

3. Roehr CC, Te Pas AB, Dold SK, et al. Investigating the European perspective of neonatal point-of-care echocardiography in the neonatal intensive care unit—a pilot study. Eur J Pediatr 2013;172:907-11.

4. Jain A, Sahni M, El-Khuffash A, Khadawardi E, Sehgal A, McNamara PJ. Use of targeted neonatal echocardiography to prevent postoperative cardiorespiratory instability after patent ductus arteriosus ligation. J Pediatr 2012;160:584-589.e1.

5. Sehgal A, McNamara PJ. Does point-of-care functional echocardiography enhance cardiovascular care in the NICU? J Perinatol 2008;28: 729-35.

6. O’Rourke DJ, El-Khuffash A, Moody C, Walsh K, Molloy EJ. Patent ductus arteriosus evaluation by serial echocardiography in preterm infants. Acta Paediatr 2008;97:574-8.

7. El-Khuffash A, Herbozo C, Jain A, Lapointe A, McNamara PJ. Targeted neonatal echocardiography (TnECHO) service in a Canadian neonatal intensive care unit: a 4-year experience. J Perinatol 2013;33:687-90.

8. Kluckow M, Jeffery M, Gill A, Evans N. A randomised placebo-controlled trial of early treatment of the patent ductus arteriosus. Arch Dis Child Fetal Neonatal Ed 2014;99:F99-F104.

9. Carmo KB, Evans N, Paradisis M. Duration of indomethacin treatment of the preterm patent ductus arteriosus as directed by echocardiography. J Pediatr 2009;155:819-822.e1.

10. Singh Y, Gupta S, Groves AM, et al. Expert consensus statement 'Neonatologist-performed Echocardiography (NoPE)'-training and accreditation in UK. Eur J Pediatr 2016;175:281-7.

11. Mertens L, Seri I, Marek J, et al.; Writing Group of the American Society of Echocardiography; European Association of Echocardiography; Association for European Pediatric Cardiologists. Targeted Neonatal Echocardiography in the Neonatal Intensive Care Unit: practice guidelines and recommendations for training. Writing Group of the American Society of Echocardiography (ASE) in collaboration with the European Association of Echocardiography (EAE) and the Association for European Pediatric Cardiologists (AEPC). J Am Soc Echocardiogr 2011;24:1057-78.

12. Mertens L, Seri I, Marek J, et al.; Targeted neonatal echocardiography in the neonatal intensive care unit: practice guidelines and recommendations for training. Eur J Echocardiogr 2011;12:715-36.

13. Lavoie PM, Stritzke A, Ting J, et al. Oral glucose during targeted neonatal echocardiography: is it useful? Arch Dis Child Fetal Neonatal Ed 2015;100:F374-5.

14. El-Khuffash AF, Jain A, Dragulescu A, McNamara PJ, Mertens L. Acute changes in myocardial systolic function in preterm infants undergoing patent ductus arteriosus ligation: a tissue Doppler and myocardial deformation study. J Am Soc Echocardiogr 2012;25:1058-67.

15. Poon CY, Edwards JM, Joshi S, Kotecha S, Fraser AG. Optimization of myocardial deformation imaging in term and preterm infants. Eur J Echocardiogr 2011;12:247-54.
16. Joshi S, Edwards JM, Wilson DG, Wong JK, Kotecha S, Fraser AG. Reproducibility of myocardial velocity and deformation imaging in term and preterm infants. Eur J Echocardiogr 2010;11:44-50.

17. Levy PT, Holland MR, Sekarski TJ, Hamvas A, Singh GK. Feasibility and reproducibility of systolic right ventricular strain measurement by speckletracking echocardiography in premature infants. J Am Soc Echocardiogr 2013;26:1201-13.

18. Czernik C, Rhode S, Helfer S, Schmalisch G, Bührer C. Left ventricular longitudinal strain and strain rate measured by 2 -D speckle tracking echocardiography in neonates during whole-body hypothermia. Ultrasound Med Biol 2013;39:1343-9.

19. Nestaas E, Skranes JH, Støylen A, Brunvand L, Fugelseth D. The myocardial function during and after whole-body therapeutic hypothermia for hypoxic-ischemic encephalopathy, a cohort study. Early Hum Dev 2014;90:247-52.

20. Wei Y, Xu J, Xu T, Fan J, Tao S. Left ventricular systolic function of newborns with asphyxia evaluated by tissue Doppler imaging. Pediatr Cardiol 2009;30:741-6.

21. Sehgal A, Wong F, Menahem S. Speckle tracking derived strain in infants with severe perinatal asphyxia: a comparative case control study. Cardiovasc Ultrasound 2013;11:34.

22. Jain A, Mohamed A, El-Khuffash A, et al. A comprehensive echocardiographic protocol for assessing neonatal right ventricular dimensions and function in the transitional period: normative data and z scores. J Am Soc Echocardiogr 2014;27:1293-304.

23. James AT, Corcoran JD, Jain A, et al. Assessment of myocardial performance in preterm infants less than 29 weeks gestation during the transitional period. Early Hum Dev 2014;90:829-35.

24. Levy PT, Dioneda B, Holland MR, et al. Right ventricular function in preterm and term neonates: reference values for right ventricle areas and fractional area of change. J Am Soc Echocardiogr 2015;28:559-69.

25. Koestenberger M, Nagel B, Ravekes W, et al. Systolic right ventricular function in preterm and term neonates: reference values of the tricuspid annular plane systolic excursion (TAPSE) in 258 patients and calculation of Z-score values. Neonatology 2011;100:85-92.

26. James AT, Corcoran JD, Franklin O, El-Khuffash AF. Clinical utility of right ventricular fractional area change in preterm infants. Early Hum Dev 2016;92:19-23.

This work is licensed under a Creative Commons Attribution-NonCommercial-NoDerivs $\quad \mathbf{4 . 0}$ International License. The images or other third party material in this article are included in the article's Creative Commons license, unless indicated otherwise in the credit line; if the material is not included under the Creative Commons license, users will need to obtain permission from the license holder to reproduce the material. To view a copy of this license, visit http://creativecommons.org/ licenses/by-nc-nd/4.0/

(C) WP de Boode et al. (2016) 